\title{
A MODELAGEM MATEMÁTICA COMO METODOLOGIA PARA O ENSINO E A APRENDIZAGEM DOS FRACTAIS
}

\author{
Marcelo Fabricio Chociai Komar - UNICENTRO - mkomar@bol.com.br \\ Dionísio Burak - PPGEN/UNICENTRO - dioburak@yahoo.com.br \\ Elaine Maria dos Santos - PPGEN/UNICENTRO - elainems77@gmail.com \\ Márcio André Martins - PPGEN/UNICENTRO - prof.mmartins@gmail.com
}

RESUMO: O presente artigo tem como objetivo relatar uma atividade de modelagem matemática a partir do tema escolhido pelos estudantes, Fractais. Participaram dessa atividade um grupo com quatro estudantes da Educação Básica, $9^{\circ}$ ano do Ensino Fundamental, no ano de 2016. Esta investigação é concebida na perspectiva qualitativa/interpretativa, com delineamento na perspectiva de Lüdke e André (1986). O tratamento dos dados segue a perspectiva de Bogdan e Biklen (1994). A linha de pesquisa em Modelagem Matemática segue os passos sugeridos por Burak (1987, 1992, 1998), Klüber e Burak (2008), para a utilização da Modelagem Matemática na perspectiva da Educação Matemática. Os resultados analisados apontam para uma melhor qualidade do ensino quando ocorre a interação e a reciprocidade entre as disciplinas.

Palavras-chave: Educação Matemática; Modelagem Matemática; Interdisciplinaridade.

ABSTRACT: The present article aims to report a mathematical modelling activity based on the students' chosen theme, Fractais. Participated in this activity a group with four students of Basic Education, 9th year of Elementary School, in the year 2016. This research is conceived in a qualitative/interpretive perspective, with a design from the perspective of Lüdke and André (1986). The treatment of the data follows the perspective of Bogdan and Biklen (1994). The research line in Mathematical Modelling follows the steps suggested by Burak (1987, 1992, 1998), Klüber and Burak (2008), for the use of Mathematical Modelling in the Mathematical Education perspective. The results analyzed point to a better quality of teaching when interaction occurs and reciprocity between the disciplines.

Keywords: Mathematical Education; Mathematical Modelling; Interdisciplinarity.

\section{Introdução}

A matemática comporta um amplo campo de relações, regularidades e coerências que despertam a curiosidade e instigam a capacidade de generalizar, projetar, prever e abstrair, favorecendo a estruturação do pensamento e o desenvolvimento do raciocínio lógico (PCN's, 1997, p.24). Também é um instrumento importante para as diferentes áreas do conhecimento, por ser utilizada em estudos ligados às ciências da natureza, assim como nas ciências sociais e também por estar presente na composição musical, na coreografia, na arte, na arquitetura, nos esportes e nos mais variados meios de expressão e comunicação (PCN's, 1997, p.25).

Referindo-se ao ensino de Matemática nos tempos atuais, pode-se observar que ainda existe, por parte do professor de Matemática, a proposição de conceber o ensino da Matemática, de forma tradicional, cartesiana e iluminista. Esta forma pode ser 
caracterizada pelo ensino como repetição e memorização dos conteúdos matemáticos, não permitindo aos estudantes vivenciar o saber matemático e conjecturar sobre sua própria realidade. Faz-se necessário, permitir ao estudante um processo de ensino e aprendizagem apropriados.

Nesse contexto a Matemática não pode ser vista como uma área isolada precisa unir-se a Psicologia, a Sociologia, a Filosofia, entre outras, de modo que possa haver uma relação coerente para o desenvolvimento do processo de ensino e aprendizagem capaz de estabelecer uma relação significativa oportunizada pela área da Educação Matemática.

A Modelagem Matemática na concepção de Burak (1987, 1992, 1998), Klüber e Burak (2008), busca pautar a Matemática a partir de situações de interesse do estudante, não como uma receita pronta para a aprendizagem, mas como um conjunto de procedimentos, envolvendo ações e interações, capazes de favorecer a formação de conceitos e a construção de conhecimentos matemáticos e outros.

Assim, o comportamento ativo do estudante, ditado pela Modelagem Matemática na concepção da Educação Matemática requer uma mudança de paradigma por parte do professor, na forma de pensar e conduzir o ensino da Matemática.

Essa mudança na maneira de conceber o ensino tem outra característica que traduz a diferença entre a Matemática e a Matemática na Educação Matemática da qual a Modelagem adita sua natureza e o arquétipo epistemológico, que direcionam suas ações e procedimentos, buscando estabelecer o conhecimento escolar do estudante, interpondo ao conhecimento científico em aula e principalmente suas características como conhecimentos sociais, fatores culturais, econômicos, entre outros.

Ao considerarmos a Modelagem Matemática como um pressuposto à problematização de situações cotidianas pode-se estabelecer um conjunto de procedimentos para o encaminhamento dos estudos dos conteúdos matemáticos, com a aceitação dos estudantes e seus conhecimentos acerca da Matemática, do social e do cultural.

Neste sentido o presente artigo procura, de maneira interdisciplinar, relatar uma das sete atividades de modelagem matemática, envolvendo quatro estudantes da Educação Básica, do $9^{\circ}$ ano do Ensino Fundamental, de um colégio público estadual, no ano de 2016, a partir do tema escolhido pelos estudantes, Fractais.

\section{Material e métodos}

Esta investigação é concebida na perspectiva qualitativa/interpretativa, com delineamento na perspectiva de Lüdke e André (1986), cujos pressupostos se apóiam em Bogdan e Biklen (1982), ao tratar das características da investigação qualitativa. Segundo Lüdke e André (1986), consiste em: (a) observar como o fenômeno de estudo é compreendido no contexto onde ocorre; (b) a descrição das atividades, como as produções dos estudantes com o tema sugerido por eles; (c) a preocupação com o processo de ensino e aprendizagem de maneira interativa; (d) nos dados pesquisados e destacar as perspectivas do trabalho no ambiente colaborativo e; (e) a análise dos dados segue um processo indutivo através da inter-relação entre professor e estudantes.

Com o intuito de iniciar o desenvolvimento dos estudos envolvendo a metodologia da Modelagem Matemática, partimos dos pressupostos presentes na concepção de Burak (1992, p.62), em que a Modelagem Matemática "constitui-se em um conjunto de procedimentos cujo objetivo é construir um paralelo para tentar explicar, matematicamente, os fenômenos presentes no cotidiano do ser humano, ajudando-o a fazer predições e tomar decisões". Ainda, adota dois princípios 1) partir 
sempre do interesse do grupo ou dos grupos participantes e 2) os dados devem ser coletados, sempre que possível, onde se dá o interesse do grupo ou dos grupos participantes.

Na sequência, foi oportunizado aos 28 estudantes que montassem 7 grupos com no máximo 4 integrantes, chamando de grupos A, B, C, D, E, F e G.

Para favorecer o encaminhamento da atividade com a modelagem matemática, foram apresentadas pelo professor aos estudantes, cinco etapas para seu desenvolvimento:

1) Escolha do tema - é o momento em que o professor apresenta aos alunos alguns temas que possam gerar interesse ou os próprios alunos sugerem um tema. Esse tema pode ser dos mais variados, uma vez que não necessita ter nenhuma ligação imediata com a matemática ou com conteúdos matemáticos, e sim com o que os alunos querem pesquisar. Já nessa fase é fundamental que o professor assuma a postura de mediador, pois deverá dar o melhor encaminhamento para que a opção dos alunos seja respeitada.

2) Pesquisa exploratória - escolhido o tema a ser pesquisado, encaminhamse os alunos para a procura de materiais e subsídios teóricos dos mais diversos, os quais contenham informações e noções prévias sobre o que se quer desenvolver/pesquisar. A pesquisa pode ser bibliográfica ou contemplar um trabalho de campo, fonte rica de informações e estímulo para a execução da proposta.

3) Levantamento dos problemas - de posse dos materiais e da pesquisa desenvolvida, incentiva-se os alunos a conjecturarem sobre tudo que pode ter relação com a matemática, elaborando problemas simples ou complexos que permitam vislumbrar a possibilidade de aplicar ou aprender conteúdos matemáticos, isso com a ajuda do professor, que não se isenta do processo, mas se torna o "mediador" das atividades.

4) Resolução dos problemas e o desenvolvimento do conteúdo matemático no contexto do tema - nessa etapa, busca-se responder os problemas levantados com o auxílio do conteúdo matemático, que pode ser abordado de uma maneira extremamente acessível, para, posteriormente, ser sistematizado, fazendo um caminho inverso do usual, pois se ensina o conteúdo para responder às necessidades surgidas na pesquisa e no levantamento dos problemas concomitantemente.

5) Análise crítica das soluções - etapa marcada pela criticidade, não apenas em relação à matemática, mas também a outros aspectos, como a viabilidade e a adequabilidade das soluções apresentadas, que, muitas vezes, são lógica e matematicamente coerentes, porém inviáveis para a situação em estudo. É a etapa em que se reflete acerca dos resultados obtidos no processo e como esses podem ensejar a melhoria das decisões e ações, contribuindo, dessa maneira, para a formação de cidadãos participativos, que auxiliem na transformação da comunidade em que participam (KLÜBER e BURAK, 2008, p.21-22).

Na sequência serão descritas as 5 etapas de acordo com a atividade Fractais.

\section{1) Escolha do Tema}

Os estudantes formados em pequenos grupos de 4 alunos sugeriram 7 temas de livre escolha: $\mathrm{O}$ uso dos computadores no laboratório de informática; Robótica; $\mathrm{O}$ uso do celular em sala de aula; A utilização do laboratório de Ciências do Colégio; Pizza; Fractais e; Projeto de leitura na biblioteca.

$\mathrm{Na}$ sequência foi sugerido aos estudantes que sorteassem um dos temas para esta pesquisa e, através de uma caixinha com pequenos papéis, elegeram como a atividade 
de modelagem matemática o tema Fractais, que ficou sobre a responsabilidade do grupo F.

\title{
2) Pesquisa exploratória
}

Os estudantes do grupo $\mathrm{F}$ pesquisaram em livros, revistas, jornais, sites de pesquisa como o Google sobre o tema Fractais. Através da pesquisa apresentaram os seguintes resultados: Geometria Fractal é o ramo da Matemática que estuda as propriedades e comportamentos dos fractais. Estão presentes nos mais variados elementos da natureza, como objetos matemáticos originários de interações geométricas contendo um mesmo padrão de construção: sucessivas divisões a partir de medidas unitárias, estas que se apresentam na forma de fração. Seus estudos foram concebidos a partir de 1975 por Benoit Mandelbrot, matemático francês nascido na Polônia (ALMEIDA, et al. 2008, p.01).

Almeida et al. (2008) consideram as principais propriedades que caracterizam e que permitem definir os conjuntos Fractais como:

\begin{abstract}
Auto-similaridade, que pode ser exata ou estatística, ou seja, mantém a mesma forma e estrutura sob uma transformação de escala (transformação que reduz ou amplia o objeto ou parte dele) (ALMEIDA, et al. 2008, p.02).

Complexidade infinita, isto é, qualquer que seja o número de amplificações de um objeto fractal, nunca obteremos a "imagem final", uma vez que ela poderá continuar a ser infinitamente ampliada (ALMEIDA, et al. 2008, p.02). Irregularidade, no sentido de rugosidade (não suavidade) ou fragmentação; Possuir em geral, dimensão não inteira. A dimensão fractal quantifica, de certo modo, o grau de irregularidade ou fragmentação do conjunto considerado (ALMEIDA, et al. 2008, p.02).
\end{abstract}

Consideram ainda que os estudos realizados no final do século XIX e início do século XX, através do uso da Geometria dos Fractais, influenciaram o estudo da geometria e possibilitaram ao homem trabalhar com as complexidades da natureza.

Como exemplo de fractais, os estudantes encontraram o Floco de Neve de Koch e o Triângulo de Sierpinski, demonstrando o interesse nesta atividade sobre o Triângulo de Sierpinski, descrito pelo matemático polonês Waclaw Sierpinski, que é obtido através da divisão de um triângulo equilátero em quatro triângulos equiláteros iguais, com sucessivas interações em seu processo de repetição. Tal interesse assumido pelos estudantes diz respeito ao conteúdo de área de figuras planas estudado do $9^{\circ}$ ano do Ensino Fundamental.

Assim decidiram pelo estudo da construção do Triângulo de Sierpinski, conforme Imagem 1:

Imagem 1: Triângulo de Sierpinski e suas interações

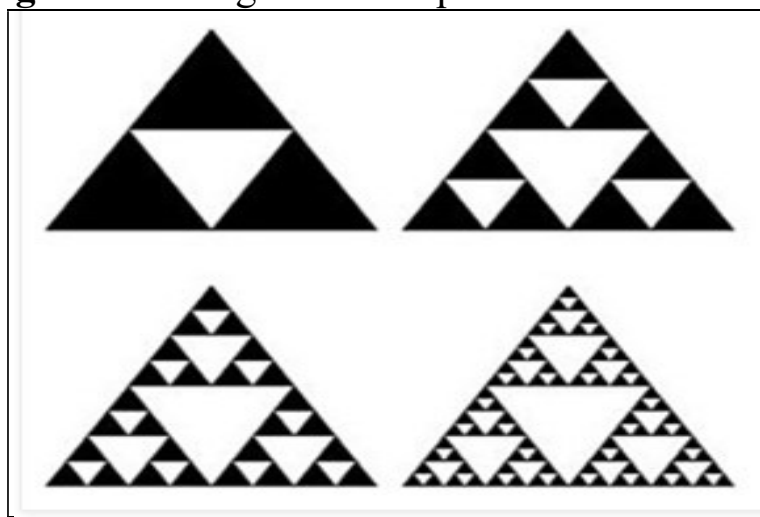

Fonte: http://professorandrios.blogspot.com.br 
$\mathrm{Na}$ sequência os estudantes do grupo $\mathrm{F}$ procuraram informações, com a mediação do professor, sobre a possibilidade de utilizar um software no Colégio, sendo sugerido o software GeoGebra, free, já instalado nos 20 microcomputadores do Colégio, que tem sistema LINUX educacional, adquiridos através dos programas Paraná Digital no ano de 2009.

\section{3) Levantamento dos problemas}

Em sala de aula, os estudantes do grupo F, responsáveis pelo tema Fractais, foram orientados a promover discussões acerca do conteúdo trabalhado e a identificar relações matemáticas possíveis com a construção do Triângulo de Sierpinski.

Assim, foi oportunizado aos estudantes do grupo $\mathrm{F}$ que elaborassem problemas, com base na pesquisa efetivada, decidindo por: Qual a expressão matemática que pode ser utilizada para construir a área do triângulo de Sierpinski com as interações, número de triângulos e novas áreas necessárias?

4) Resolução dos problemas e o desenvolvimento do conteúdo matemático no contexto do tema

Após levantamento dos problemas, os estudantes foram conduzidos ao Laboratório de Informática, e com a mediação do professor e o auxílio do software GeoGebra, procedemos inicialmente com a verificação do conhecimento prévio dos estudantes do grupo F, frente à ferramenta, a Interface do software GeoGebra, que se mostraram entusiasmados com a utilização da tecnologia, conforme descreve a Imagem 2:

Imagem 2: Interface do software GeoGebra

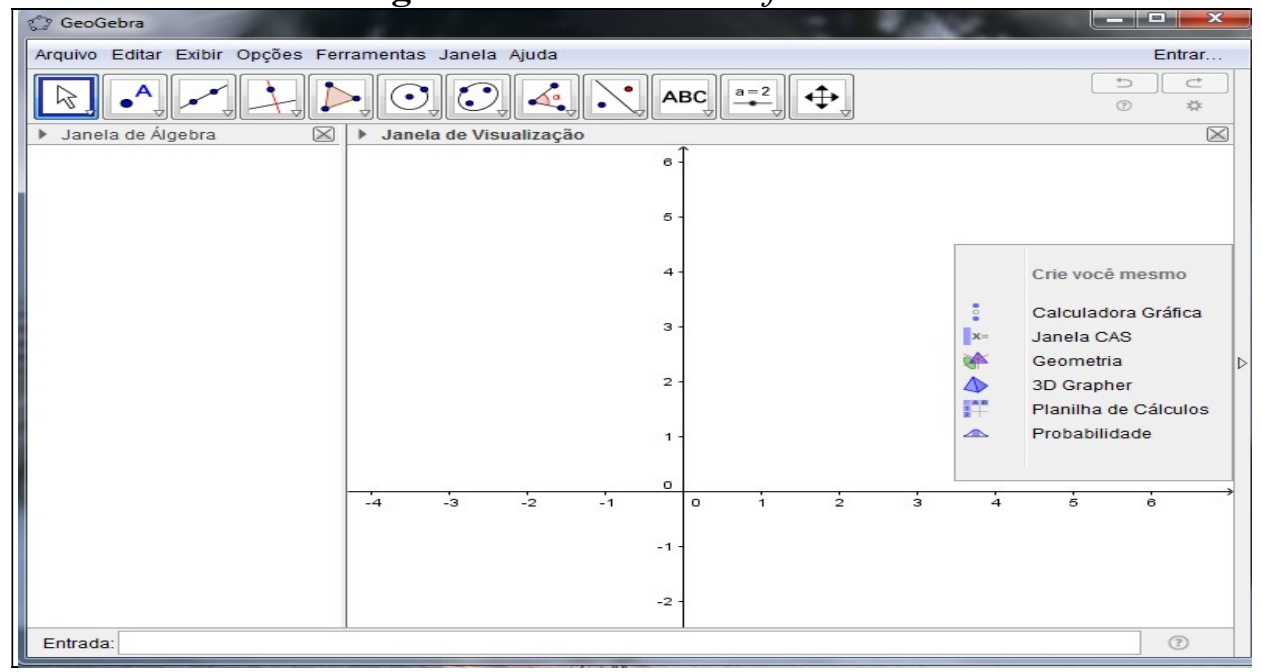

Fonte: Estudantes - 2016

Para a construção do Triângulo de Sierpinski, os estudantes do grupo $\mathrm{F}$ atribuíram a função polígono, presente na barra de ferramentas do software GeoGebra, como mostra a Imagem 3: 
Imagem 3: Ferramenta Polígono disponível no software GeoGebra

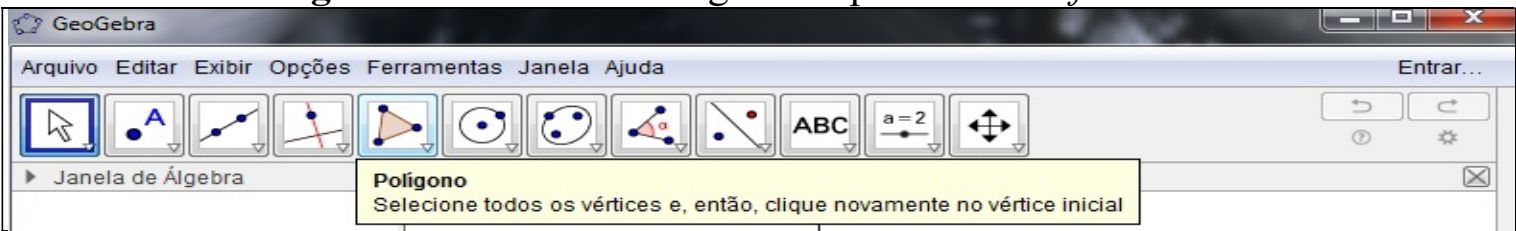

Fonte: Estudantes - 2016

Esta etapa, mencionada por Almeida et al. (2008), identifica o estudo da divisão de um triângulo equilátero em quatro triângulos equiláteros iguais, com seu centro retirado e após sucessivas interações seu processo sendo repetido, o que caracterizou a seguinte construção:

$1^{\circ}$ Os estudantes abriram uma janela no GeoGebra escondendo os eixos e a malha quadriculada, com a opção exibir eixos e malhas;

$2^{\circ}$ Construíram um triângulo equilátero utilizando o ícone Polígono (Imagem 3);

$3^{\circ}$ Marcaram (A, B, C) e os pontos médios utilizaram o segundo ícone, como mostra a Imagem 4:

Imagem 4: Ponto

\begin{tabular}{|ll|l|l|l|l|l|}
\hline 2 GeoGebra & $x$ \\
\hline Arquivo Editar Exibir Opções Ferramentas Janela Ajuda
\end{tabular}

Fonte: Estudantes - 2016

$4^{\circ} \mathrm{Na}$ sequência criaram um novo triângulo equilátero tendo como base $\mathrm{o}$ triângulo equilátero já construído;

$5^{\circ}$ Desconsideram o triângulo central, obtido pelos passos anteriores, marcandose os pontos médios de cada um dos lados dos demais triângulos que foram unidos pelos segmentos, desconsiderando o triângulo do meio, sucessivamente, chegando assim no resultado esperado. Concluíram, com a mediação do professor, que ao clicar na opção ferramentas - criar nova ferramenta, as seguintes condições: objetos finais: quando clicam nos segmentos com o botão esquerdo do mouse e definem-se os objetos; objetos iniciais: que são os objetos informados no início do trabalho e por fim criam-se o nome e ícone, que será definido como Triângulo de Sierpinski e que servirá para construir quantas interações forem necessárias, como mostra a Imagem 5a:

Imagem 5a: Primeira interação para a construção do Triângulo de Sierpinski

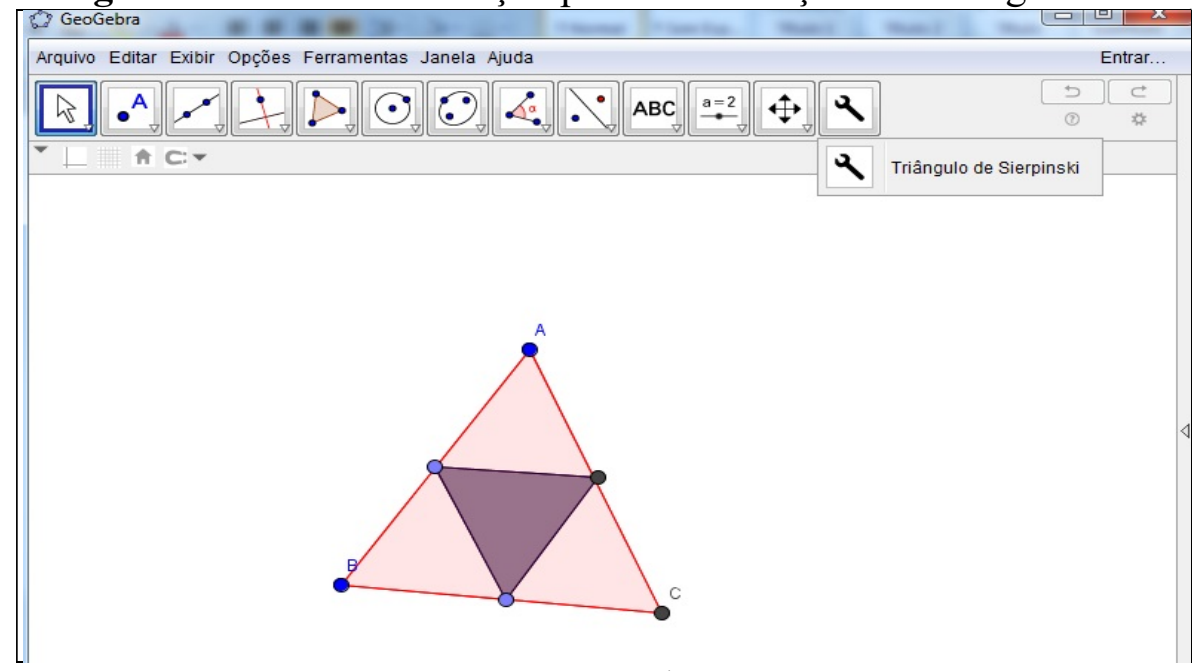

Fonte: Estudantes - 2016 
Com base na Imagem 5(a), coube aos estudantes do grupo $\mathrm{F}$ esclarecer matematicamente a área do triângulo encontrada pelo software, assim encontraram a seguinte expressão matemática da área inicial do triângulo apresentado (1):

$$
A=\frac{l^{2} \sqrt{3}}{4}
$$

Como descrito pelo matemático polonês Waclaw Sierpinski, consideraram ainda em (1) que cada triângulo equilátero é divido em quatro triângulos equiláteros iguais, ou seja, uma expressão genérica do tipo (2):

$$
A=\frac{l^{2} \sqrt{3}}{4.4^{n}}
$$

Em que $n$ identifica o número das interações

Assim, após várias interações com o software, os estudantes do grupo $\mathrm{F}$ chegaram a seguinte Imagem $5 \mathrm{~b}$ :

Imagem 5b: Várias interações para a construção do Triângulo de Sierpinski

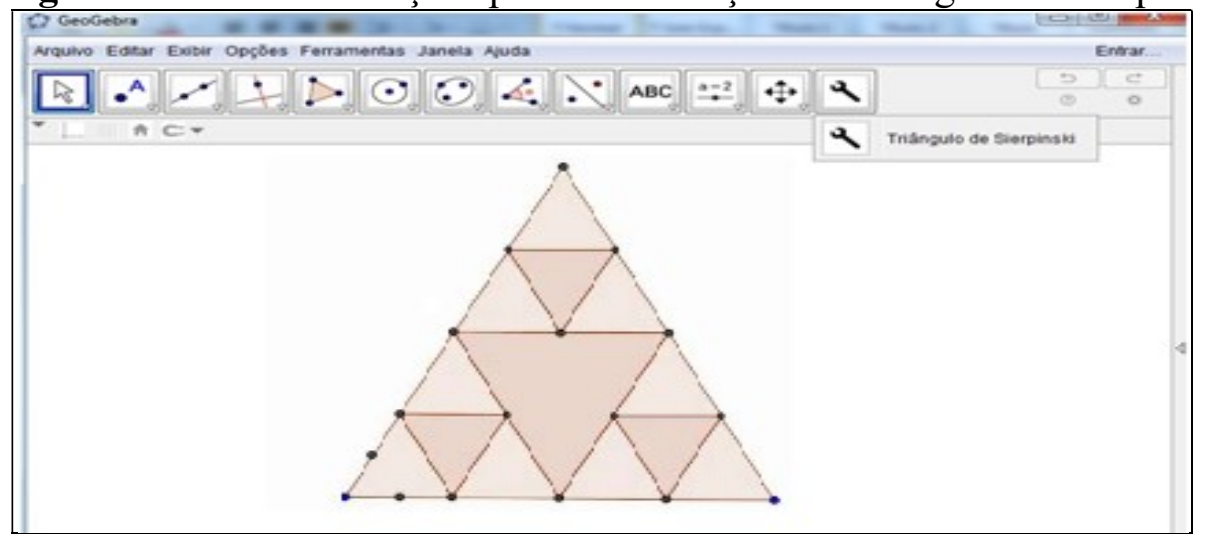

Fonte: Estudantes - 2016

Com base na Imagem 5(b), coube aos estudantes do grupo $F$ esclarecer matematicamente se a expressão genérica (2), a partir de várias interações para a construção do Triângulo de Sierpinski, estaria correta, chegando nas seguintes considerações conforme descreve o Quadro 1:

Quadro 1: Interações entre número de triângulos retirados e área novo triângulo

\begin{tabular}{|c|c|l|}
\hline Interações & $\begin{array}{c}\text { Numero de triângulos } \\
\text { Retirados }\end{array}$ & $\begin{array}{c}\text { Área novo } \\
\text { Triângulo }\end{array}$ \\
\hline 0 & 1 & $A=\frac{l^{2} \sqrt{3}}{16.4^{0}}$ \\
\hline 1 & 3 & $A=\frac{l^{2} \sqrt{3}}{16.4^{1}}$ \\
\hline 2 & 9 & $A=\frac{l^{2} \sqrt{3}}{16.4^{2}}$ \\
\hline 3 & 27 & $A=\frac{l^{2} \sqrt{3}}{16.4^{3}}$ \\
\hline------- & ------- & --------- \\
\hline$n$ & $3^{n}$ & $A=\frac{l^{2} \sqrt{3}}{16.4^{n}}$ \\
\hline
\end{tabular}

Fonte: Estudantes - 2016 
Assim, os estudantes comprovaram através das expressões matemáticas, a fórmula de uma área genérica para um novo Triângulo de Sierpinski.

\title{
5) Análise crítica das soluções
}

Com base nos diálogos das estudantes, sobre as questões que dizem respeito à solução dos problemas levantados, foram feitas observações, comentários e discussões relativas a atividade Fractais.

Uma situação que ensejou uma análise crítica foi a relação com o conceito de auto semelhança, citado por Almeida et al. (2008), em que os estudantes puderam perceber as seguintes relações entre ramos de cedro e hortaliças, como objetos de estudos das disciplinas de Ciências e Biologia, mostrando a natureza interdisciplinar da pesquisa, apresentando estes elementos conforme mostra a Imagem 6.

Imagem 6: semelhança aproximada

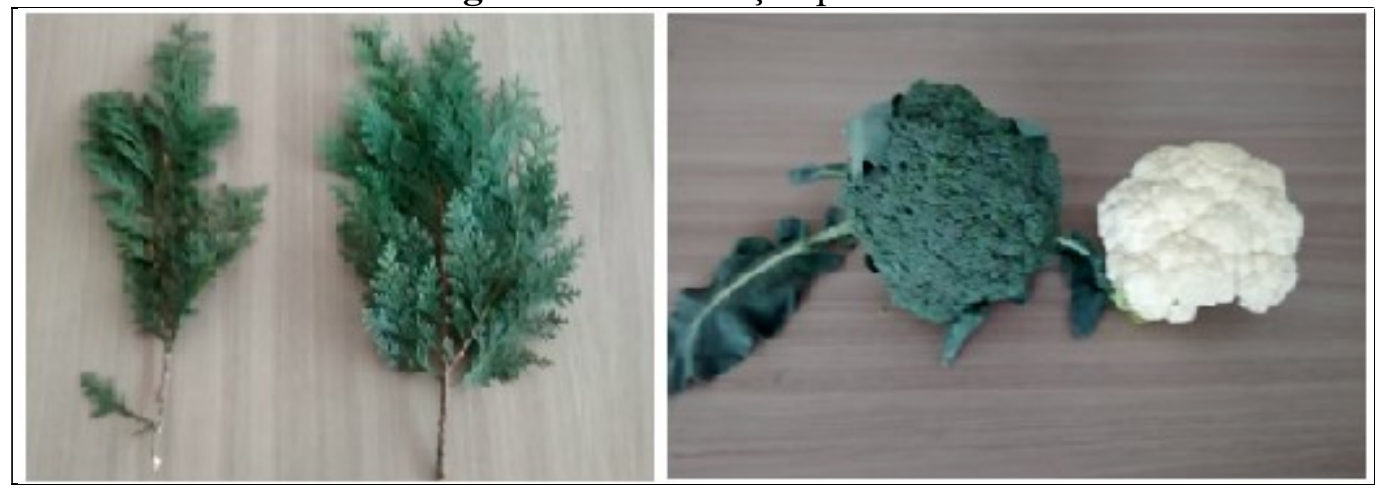

Fonte: Estudantes - 2016

As questões relativas aos conteúdos matemáticos, como o conceito da área, razão e proporcionalidade do Triângulo de Sierpinski foram bem associadas pelos estudantes, após a utilização da TIC computador e software GeoGebra, sendo perceptível a condução da atividade de maneira correta, com a mediação adequada do professor. Valente (1993) cita que: "a mudança da função do TIC computador como meio educacional acontece juntamente com um questionamento da função da escola e do papel do professor". Para o autor:

\begin{abstract}
a verdadeira função do aparato educacional não deve ser a de ensinar, mas sim a de criar condições de aprendizagem. Isso significa que o professor precisa deixar de ser o repassador de conhecimento - o computador pode fazer isso e o faz tão eficiente quanto professor - e passar a ser o criador de ambientes de aprendizagem e o facilitador do processo de desenvolvimento intelectual do aluno (VALENTE, 1993, p. 06).
\end{abstract}

Para consolidar a aprendizagem, os estudantes ainda relacionaram os estudos do software GeoGebra na construção do Triângulo de Sierpinski, com as expressões matemáticas que dão sustentação aos cálculos de área dos triângulos e inclusive uma forma genérica para obtenção de uma nova área com $n$ interações. Esta mediação com o estudante deve ser tratada com muita responsabilidade, pois é admitida como "verdadeira e segura" (VYGOTSKY, 1991, p.89).

Ainda, em relação aos estudantes pôde-se perceber durante a realização das atividades, o entendimento do comprometimento com a aprendizagem, o que nos faz 
acreditar ainda mais que "para ser interdisciplinar é preciso ter um conhecimento complexo" e "o pensamento complexo nos diz que o conhecimento nunca estará completo e que a racionalidade tem limites" (MORIN, 2007, pp.25-27).

Esse comprometimento segundo Piaget (1982) apresenta a interação e reciprocidade cognitivos entre as Ciências, numa perspectiva transdisciplinar, ou seja, a forma de pensar descrita pelos estudantes.

\section{Resultados e discussões}

As relações obtidas com a metodologia da Modelagem Matemática, a partir do tratamento das informações oportunizou aos estudantes, através do conceito de auto semelhança, citado por Almeida et al. (2008), observar estruturas reais na natureza que possam dar sustentação a teoria dos Fractais, o que pode ser gratificante em nosso estudo, assim puderam inter-relacionar a semelhança com ramos de cedro, o brócolis, a couve-flor. Tal conceito pode ser evidenciado por Vygostky (1998) apud Richit (2004) a zona de desenvolvimento proximal, que se refere à "região" ou "distância" entre aquilo que o aluno já sabe, que já foi assimilado, isto é, distância entre seu desenvolvimento real, que se costuma determinar através da solução independente de problemas e o nível de seu desenvolvimento potencial, determinado através da solução de problemas sob a orientação de um adulto ou em colaboração com companheiros mais capazes.

A interação com as TIC demonstra a importância da inter-relação com outras áreas do conhecimento, através dos conceitos abordados por Burak e Klüber (2011), que a Matemática parte de situações de interesse do estudante, não como uma receita pronta para a aprendizagem, mas como um conjunto de procedimentos, envolvendo ações e interações capazes de favorecer a formação de conceitos e a construção de conhecimentos matemáticos e outros.

\section{Conclusões}

Este trabalho oportunizou o relato e a discussão da atividade de modelagem matemática desenvolvida a partir do tema: Fractais, vivenciando a metodologia da Modelagem Matemática e o envolvimento interdisciplinar com outras áreas do conhecimento.

Os procedimentos dessa investigação foram conduzidos na perspectiva qualitativa/interpretativa, com delineamento de Lüdke e André (1986), cujos pressupostos se apóiam em Bogdan e Biklen (1982), apontaram neste estudo, a qualidade do ensino quando tratado de forma interdisciplinar, em que a Matemática não deve ser vista como uma ciência isolada, mas que possa promover o processo de ensino e aprendizagem aos estudantes de maneira significativa, com intuito de promover a possibilidade de formação humana, crítica e reflexiva no estudante.

Assim, o conhecimento presente nos aspectos epistemológicos, orientado e favorecido pela natureza da Educação Matemática, pode diferenciar características das teorias de aprendizagem presentes no âmbito da escola e outros fundamentos importantes da educação, como o diálogo, o interesse do estudante, sobre o ser do estudante como um sujeito único, reconhecendo a importância de suas experiências anteriores e, potencializado pela utilização da Modelagem Matemática como metodologia de ensino, cujos fundamentos tornam o ambiente escolar colaborativo, permitindo visualizar com clareza um processo de ensino com vistas à aprendizagem. 


\section{Referências}

ALMEIDA, T. B.; MARTINELLI, R. O.; RODRIGUES, V. M.; SilVA, A. M. M. Fractais no Ensino Fundamental: explorando essa nova geometria. Disponível em $<$ www.sbembrasil.org.br/files/ix_enem/Poster/.../PO00995663033T.doc > Acesso em 20 de setembro de 2015.

BIKLEN, S; BOGDAN, R. C.. Investigação qualitativa em educação. Porto: Porto Editora, p. 134-301, 1994.

BURAK, D. Modelagem Matemática: uma metodologia alternativa para o ensino de matemática na $5^{\text {a }}$ série. Rio Claro-SP, 1987. Dissertação (Mestrado em Ensino de Matemática) - IGCE, Universidade Estadual Paulista Júlio Mesquita Filho-UNESP.

BURAK, D. Modelagem Matemática: ações e interações no processo de ensinoaprendizagem. 1992. 460p. Tese (Doutorado em Psicologia Educacional). Universidade Estadual de Campinas Faculdade de Educação. SP.

KLÜBER. T. E.; BURAK, D. Concepções de modelagem matemática: contribuições teóricas. In: Educação Matemática e Pesquisa., São Paulo, v.10, n.1, pp-17-34, 2008.

LÜDKE, M e ANDRÉ, M. E. D. A.. Pesquisa em educação: abordagens qualitativas. São Paulo: EPU, 1986.

MORIN, Edgar. Desafios da transdisciplinaridade e da complexidade. In: AUDY, Jorge Luis Nicolas; MOROSINI, Marília Costa (Orgs). Inovação e interdisciplinaridade na Universidade. Porto Alegre: Edipucrs, 2007.

Parâmetros Curriculares Nacionais: Matemática. Secretaria de Educação Fundamental. - Brasília. MEC/SEF, 1997. Disponível em < http://portal.mec.gov.br/seb/arquivos/pdf/livro03.pdf> Acesso em 01 de maio de 2016.

PIAGET, J. \& INHELDER, B. A Psicologia da Criança. São Paulo: Difel. 1982.

RICHIT, A. Implicações da teoria de Vygotsky aos processos de aprendizagem e desenvolvimento em ambientes mediados pelo computador. Revista Perspectiva, Erechim, RS, v. 28, n. 103, p. 21-32, 2004.

Uma experiência com a Modelagem Matemática. PRÓ-MAT, Curitiba, v.1, p.32-47. 1998.

VALENTE, J. A. Computadores e conhecimento: repensando a educação. Campinas: UNICAMP. 1993. 